\title{
TargetCOPD: a pragmatic randomised controlled trial of targeted case finding for COPD versus routine practice in primary care: protocol
}

\author{
Rachel E Jordan ${ }^{1}$, Peymané Adab ${ }^{1,5^{*}}$, Sue Jowett ${ }^{1}$, Jen L Marsh', Richard D Riley ${ }^{1}$, Alexandra Enocson ${ }^{1}$, \\ Martin R Miller ${ }^{1}$, Brendan G Cooper ${ }^{2}$, Alice M Turner ${ }^{3}$, Jon G Ayres ${ }^{1}$, Kar Keung Cheng ${ }^{1}$, Kate Jolly ${ }^{1}$, \\ Robert A Stockley ${ }^{2}$, Sheila Greenfield ${ }^{1}$, Stanley Siebert ${ }^{4}$, Amanda Daley ${ }^{1}$ and David A Fitzmaurice ${ }^{1}$
}

\begin{abstract}
Background: Many people with clinically significant chronic obstructive pulmonary disease (COPD) remain undiagnosed worldwide. There are a number of small studies which have examined possible methods of case finding through primary care, but no large RCTs that have adequately assessed the most cost-effective approach.

Methods/Design: In this study, using a cluster randomised controlled trial (RCT) in 56 general practices in the West Midlands, we plan to investigate the effectiveness and cost-effectiveness of a Targeted approach to case finding for COPD compared with routine practice. Using an individual patient RCT nested in the Targeted arm, we plan also to compare the effectiveness and cost-effectiveness of Active case finding using a postal questionnaire (with supplementary opportunistic questionnaires), and Opportunistic-only case finding during routine surgery consultations. All ever-smoking patients aged 40-79 years, without a current diagnosis of COPD and registered with participating practices will be eligible. Patients in the Targeted arm who report positive respiratory symptoms (chronic cough or phlegm, wheeze or dyspnoea) using a brief questionnaire will be invited for further spirometric assessment to ascertain whether they have COPD or not. Post-bronchodilator spirometry will be conducted to ATS standards using an Easy One spirometer by trained research assistants.

The primary outcomes will be new cases of COPD and cost per new case identified, comparing targeted case finding with routine care, and two types of targeted case finding (active versus opportunistic). A multilevel logistic regression model will be used to model the probability of detecting a new case of COPD for each treatment arm, with clustering of patients (by practice and household) accounted for using a multi-level structure.

A trial-based analysis will be undertaken using costs and outcomes collected during the trial. Secondary outcomes include the feasibility, efficiency, long-term cost-effectiveness, patient and primary care staff views of each approach.
\end{abstract}

Discussion: This will be the largest RCT of its kind, and should inform how best to identify undiagnosed patients with COPD in the UK and other similar healthcare systems. Sensitivity analyses will help local policy-makers decide which sub-groups of the population to target first.

Trial registration: Current controlled trials ISRCTN14930255

Keywords: COPD, Case-finding, Screening, Primary care, Respiratory questionnaire, Spirometry, Cluster RCT, Cost-effectiveness

\footnotetext{
* Correspondence: p.adab@bham.ac.uk

'School of Health and Populations Sciences, University of Birmingham, Birmingham, UK

${ }^{5}$ Public Health, Epidemiology \& Biostatistics, Public Health Building, School of Health and Population Sciences, University of Birmingham, Edgbaston, Birmingham B15 2TT, UK

Full list of author information is available at the end of the article
} 


\section{Background}

Chronic obstructive pulmonary disease (COPD) affects $5-10 \%$ of adults worldwide [1], is rising in prevalence [2], and is a leading cause of death [3]. In the UK it accounts for 1.4 million GP consultations, 1 million in-patient days per year and costs the NHS over $£ 800$ million per year [4]. However, around $45-85 \%$ of cases [5-7], depending on diagnostic criteria, remain undiagnosed, representing many with potentially unmet need. There is much uncertainty about how to approach early identification of patients $[8,9]$, although it has been recently demonstrated that there are many missed opportunities to diagnose patients in primary care where signs and symptoms have been overlooked [10].

The CMO's report, 2005 [11] and the Healthcare Commission report [12] highlighted the burden of COPD, the extent of under-diagnosis and variation in access to relevant services. As a result a National Clinical Outcomes Strategy has been published recommending case finding in high risk patients [13], resulting in a British Lung Foundation campaign [14] and drive to identify missing cases $[15,16]$. Several NHS Trusts have started initiatives $[17,18]$.

Despite the move towards early case-finding for COPD, the most effective and cost-effective approach is not known. There are no relevant published systematic reviews, though many small pre-post studies have reported on approaches to case finding. These report either active $[5,19,20]$ or opportunistic [21-24] approaches (with yield 18-47\%), but are limited because of a lack of comparison groups, restricted number and range of participants, incomplete follow up or different target populations. More recently a few trials have been published. A small trial from a single practice reported a higher prevalence of airflow obstruction with an opportunistic compared to invitation based approach to case finding [25]. The population however was highly deprived, with many non-English speakers, and no economic analysis was undertaken. A cluster RCT in 16 general practices in the Netherlands which used an initial screening questionnaire to identify patients at risk found that a practicemanaged approach to calculating the score and inviting patients for spirometry assessment was more effective than a patient-led approach of applying for spirometry assessment having calculated their own scores [26]. Additionally, we have also reported a pilot study in two general practices which suggested that it may be more cost-effective to undertake opportunistic screening; however the study was underpowered and the results require proper testing in a full RCT [27]. The limitation of the current evidence base and lack of adequate data from RCTs contributed to the recent recommendation by the UK National Screening Committee against screening for COPD [28], and justifies a new adequately-powered trial.

Many studies have used spirometry alone to screen for COPD. However this is not recommended [8] as it would identify many without clinically important disease for whom there is little evidence of effective interventions $[29,30]$. National Institute for Clinical Excellence (NICE) guidelines recommend opportunistic case finding in those presenting with relevant symptoms and exposures (mainly smoking) [31]. Our analysis of the Health Survey for England (HSE), a large dataset representative of the English population, shows that targeted case-finding among smokers aged $\geq 40$ with relevant symptoms would have greater yield than targeting the general population [32]. Of the undiagnosed cases likely to be detected, more than three quarters could benefit from evidence based treatment (e.g. pulmonary rehab, inhaled therapies, vaccination \& smoking cessation interventions), and there is new evidence that others may also benefit [33]. Modelling of data from the Health Survey for England indicated that active case-finding (with postal questionnaire plus opportunistic case-finding in primary care consultations) would be more effective than opportunistic case-finding alone, but this needs empirical testing [32].

Cases identified are expected to benefit from treatment resulting in improved quality of life, increased survival, reduction in hospital admissions and less work-related absence. Published economic evaluations in COPD have primarily considered interventions for the disease rather than diagnosis, and others have concentrated on the costs of COPD in burden of illness studies. No trial-based economic evaluation has considered case-finding. NICE guidelines included simple decision-tree based modelling to determine the cost-effectiveness of opportunistic case finding among ever smokers over 35 years old with chronic cough [31]. The model is simplistic, contains many assumptions and requires better data on the natural history of the disease. The proposed cost-effectiveness component of this study will resolve this issue.

In this study, using a cluster RCT in general practices across the West Midlands, we plan to investigate the effectiveness and cost-effectiveness of a Targeted approach to case finding for COPD compared with routine practice. Using an individual patient RCT nested in the targeted arm, we plan also to compare the effectiveness and cost-effectiveness of Active case finding using a postal questionnaire (with supplementary opportunistic questionnaires), and Opportunistic case finding at routine surgery consultations.

\section{Methods}

\section{Aims and objectives}

- To determine whether Targeted case finding is more effective and cost-effective than routine practice.

- To evaluate the relative effectiveness and cost-effectiveness of two alternative methods of targeted case finding: Active (postal questionnaire 
plus flagging of patient records) versus Opportunistic (flagging only).

\section{Design}

TargetCOPD is a pragmatic randomised controlled trial of a case-finding intervention, delivered at organisational level, with two elements (Figure 1):

1. Cluster randomised trial comparing Targeted case finding ( 28 practices) with routine practice ( 28 practices).
2. Individually randomized controlled trial nested within the targeted case finding arm comparing two alternative types of targeting: Active (A) versus Opportunistic (O).

\section{Setting}

Fifty six general practices in the West Midlands will be recruited through the Primary Care Research Network for Central England (PCRN-CE) at the University of Birmingham.

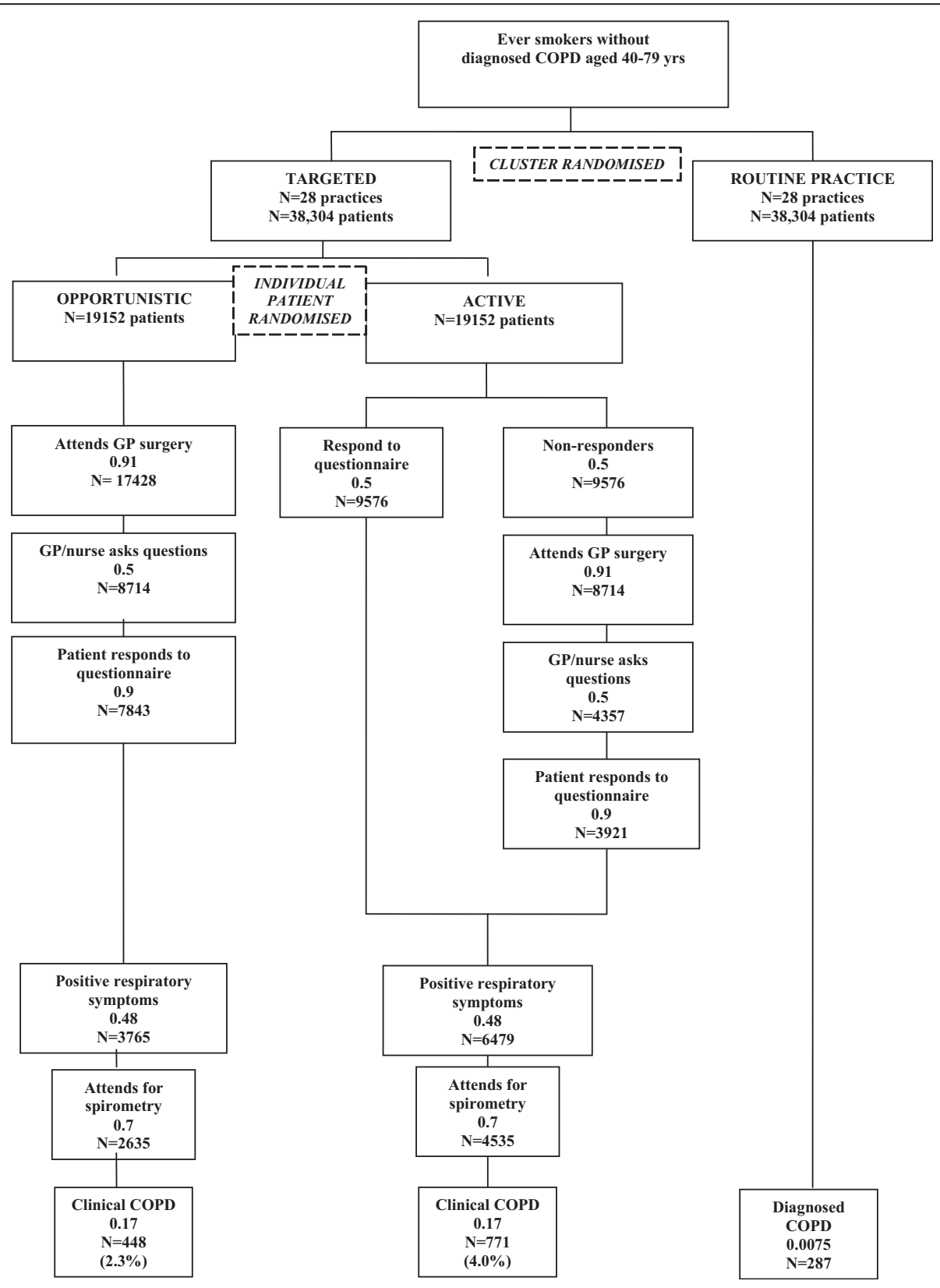

Figure 1 Plan of trial including projected numbers [32]. 


\section{Participants}

Patients will be identified as eligible if they are:

- registered at a participating practice

- aged between 40 and 79 years (inclusive) on the date of the record search,

- identified as current or ex-smokers according to their patient records

- have no prior diagnosis of COPD

Automated computer searches will be used for identifying all eligible patients using READ codes (Additional file 1: Appendix 1). Patients will be further assessed for eligibility to take part in assessments by their GP and those not considered suitable will not be invited for assessment (e.g. if unable to give informed consent, suffering from a terminal illness or pregnant).

\section{Intervention}

All interventions will take place over one year. The schema is given in Figure 1 with projected patient numbers. Fifty six practices will initially be randomised to continue with routine care or to a targeted approach to case finding.

\section{Routine practice arm}

In the routine practice arm of the cluster trial, practices will continue with their usual care and new cases of COPD will be identified according to usual practice. NICE guidance recommends that patients over the age of 35 years should be investigated for COPD with spirometry if they present opportunistically with chronic cough or phlegm [31].

\section{Targeted case finding arm}

Within the targeted arm of the cluster trial, participants will be randomly allocated (by household) to receive either the Opportunistic or the Active case finding intervention:

\section{Stage 1: respiratory questionnaire}

Opportunistic Patients in this arm will have their records flagged to prompt the consulting healthcare practitioner or receptionist to provide them with a brief screening questionnaire consisting of simple respiratory questions supplemented with basic socio-demographic and medical information (Additional file 1: Appendix 2). The questionnaire is based on validated questions, where available, and has been piloted. Patients who agree to take part will leave the questionnaire with the receptionist.

Active In addition to having their records flagged to allow opportunistic provision of the questionnaire, eligible patients in the Active arm will also be sent a postal questionnaire (with reply-paid envelope). If the questionnaire has not been received within four weeks after posting, then a first reminder will be sent. After a further four weeks, a second reminder will be sent. (It has been shown that the response rate to respiratory questionnaires is maximized with two reminders) [34].

Once the questionnaire has been returned to the investigators, the computer flag will be removed.

\section{Stage 2: spirometry assessment}

Any patient responding positively to the respiratory symptom questions (chronic cough or phlegm for three or more months of the year for two or more years, wheeze in the last 12 months or dyspnoea of MRC grade 2 or more) will be invited to attend for spirometry to ascertain whether they have COPD or not.

This will take place in a room at the patient's GP practice or an alternative local venue. Post-bronchodilator spirometry will be undertaken according to ATS/ERS 2005 guidelines [35] and carried out by research assistants trained to high standards using a short modified programme modelled on the ARTP Spirometry course by the lung function unit at Queen Elizabeth Hospital Birmingham. The research assistants will undertake frequent refresher training and monitoring of quality throughout the trial. $\mathrm{FEV}_{1}, \mathrm{FVC}, \mathrm{FEV}_{6}$ will be measured using the Easy One spirometer (ndd, Switzerland). Patients will be administered 400 micrograms salbutamol through a spacer, and asked to rest for 20 minutes prior to undergoing spirometry. Customised software (MMiller) will be used to ensure real-time quality of each manoeuvre and every trace over-read.

All spirometry results will be provided to their GP.

During the assessment visit, patients will also be asked to complete a short questionnaire to ascertain their out of pocket expenses for attendance (Additional file 1: Appendix 3), health status (EQ-5D) [36], job type (using CASCOT software) and the COPD Assessment Test. [37] Patients' height will be measured to the nearest $\mathrm{cm}$ using a portable stadiometer (or estimated using arm-span if necessary), and weight (to the nearest $0.1 \mathrm{~kg}$ ) will also be measured using GP scales by trained researchers.

\section{Allocation to trial arm \\ Cluster randomised trial comparing targeted case finding with routine care}

Recruited practices will be allocated into intervention arms using block randomization. Ideally block size will be at least eight practices $[38,39]$, with an equal number of practices allocated to intervention and control groups by the end of each block.

A minimization algorithm will be used to allocate practices to intervention groups to ensure balance across the groups for the following covariates: deprivation, ethnicity, practice size, age and proportion of COPD patients on the Quality and Outcomes Framework (QOF) 
COPD register [40]. Any linked practices (those practices that have the same GPs across two sites, each with a different patient list) will be stratified so that the allocation to trial arm will be the same in order to avoid contamination. The algorithm generates an ordered list of the possible permutations of randomization within each block - at the top of the list are the permutations which minimize imbalance the best. Of those at the top of the list (e.g. the top 10\%), one will be chosen at random to use.

\section{Individually randomised RCT comparing active with opportunistic case finding}

Within each practice randomised to the Targeted arm, each household will be further randomised to either active or opportunistic case finding. Thus in the event of multiple patients from the same household, they will therefore be allocated the same intervention group in order to avoid contamination. Allocation will be undertaken automatically by the research database as each practice list is uploaded.

\section{Outcomes}

\section{Primary outcomes}

The primary outcomes are the percentage of the eligible population diagnosed as new COPD cases within the first year, and cost per additional case identified in each arm, comparing:

1. Targeted case finding and routine practice

2. Active and opportunistic case finding

\section{Secondary outcomes}

1. Feasibility (process measures such as uptake and resource needs).

2. Efficiency (number needed to target to identify one person likely to benefit).

\section{Diagnosing COPD}

For the cluster part of the trial, patients in the routine arm may only be newly diagnosed with COPD during usual care and will be identified from GP records using the same method as that used to identify and exclude patients with a prior diagnosis of COPD in the initial search (Additional file 1: Appendix 1). The current NICE guidance is that patients with appropriate chronic respiratory symptoms must also meet spirometric criteria $\left(\mathrm{FEV}_{1} / \mathrm{FVC}<0.7\right)$ [31]. Thus, in order to compare with routine care, our primary definition must be $\mathrm{FEV}_{1} / \mathrm{FVC}$ $<0.7$ in the presence of respiratory symptoms. However this definition is controversial, and therefore, we propose also to use the "Lower Limit of Normal" (LLN) definition using the recently established GLI 2012 equations
[41]. This requires that the post-bronchodilator $\mathrm{FEV}_{1} /$ FVC ratio falls below the 5th percentile of the predicted values for patients' age, height, sex and ethnicity. All patients must also report chronic respiratory symptoms. In sensitivity analyses we will explore other definitions such as use of different cut-offs for the LLN.

\section{Additional data collection}

Data on the practices and patients will be collected at several stages during the trial:

\section{Practice level information}

Information on the characteristics of each of the participating practices will be collected, to include list size, number of eligible patients, number of existing COPD patients, deprivation score, rural/urban status, ethnicity profile, number of GPs, designated practice contacts and age profile of the practice.

\section{Information on all eligible patients}

At baseline, practices will provide an anonymised dataset on each of the eligible patients (in both routine practices and targeted arms) to allow comparison between the arms of the trial. To include: age, sex, ethnicity, smoking status, co-morbidities, health service use and reasons for exclusion.

At the end of the year, practices will also provide information on the number of consultations for each eligible patient, number of new cases of COPD identified outside or within the trial, whether patients have left the practice or have died, and among a sample, the number of flags remaining on the GP system to give an indication of the proportion of questionnaires distributed opportunistically.

\section{Statistical justification for sample size}

As there are two primary outcomes, the significance level for multiple testing is adjusted so that in total (across both sample size calculations) there is a 5\% significance level. The individually randomised component (outcome (b) below) comparing Active versus Opportunistic case finding requires far fewer patients for adequate power and is therefore given $0.25 \%$ significance level. The cluster component (a) is designated $4.75 \%$ significance level.

\section{(a) Targeted case finding versus Routine care cluster randomized design}

Based on the authors' modelling work [32], the proportion of new COPD cases detected in the Targeted group (averaged across both Active and Opportunistic arms) was assumed to be $3.15 \%$. Based research by Simpson et al. [42], the proportion of new COPD cases detected in the Routine care group was assumed to be $0.75 \%$. At 
the $4.75 \%$ significance level, with $80 \%$ power, this led to an unadjusted sample size required of 545 per group, in order to detect a difference of $2.4 \%$ between Targeted and Routine care arms.

We expect $\sim 40 \%$ of a practice population to be age 40-79 yrs with $57 \%$ of these being ever smokers without a previous diagnosis of COPD [32]. Assuming therefore a conservative 1000 eligible patients per practice of average list size 6000, and adjusting for clustering of patients within practices, assuming a conservative ICC of 0.05 $[43,44]$ the sample size required was 27,768 per arm, equivalent to 28 practices per arm.

\section{(b) Targeted arm: Active versus opportunistic case finding}

The following estimates are based on assumptions drawn from the authors' previous modelling work [32]. We assume $50 \%$ allocated to the Active arm will respond; of the remaining patients $91 \%$ will visit their GP at least once in 12 months, 50\% are offered the questionnaire and $90 \%$ of these will fill it out. In the Opportunistic arm we assume $50 \%$ are offered the questionnaire and of these $90 \%$ complete questionnaires (Figure 1). Of all responders to the questionnaire in both arms, we assume $48 \%$ are likely to report symptoms and be invited to spirometry, of which $70 \%$ will attend and $17 \%$ of these will have COPD. This leads to yield of $2.3 \%$ in the Opportunistic and $4.0 \%$ in the Active arms. At a $0.25 \%$ significance level, 3904 patients/arm are required to detect this difference with $90 \%$ power.

For the targeted case finding comparison, there will be 19,152 eligible in each arm, giving ample power for the primary comparison (based on above assumptions 448 new cases from Opportunistic arm and 771 from Active arm). This sample size provides additional power for secondary analyses and also allows more flexibility should the assumptions be more optimistic than anticipated.

\section{Statistics and data analyses}

All statistical analyses will be undertaken in STATA version 13.

\section{Primary analyses}

There are two primary comparisons:

\section{Targeted case finding vs Routine care (cluster design)}

Baseline characteristics of patients and uptake rate at each stage will be described for each cluster, and compared across clusters to check for balance across groups. A multilevel logistic regression model will be used to model the probability of detecting a new case of COPD in the Targeted group compared with the Routine care group. Clustering of patients (by practice and household) will be accounted for using a multi-level structure. The data will also be modelled using a log-link and results from the logit-link and log-link models will be compared. OR and also RR and risk differences will be estimated $[45,46]$, as will the NNT (Number Needed to Target to identify one additional COPD case). The analysis will also adjust for variables used in the randomization (age, ethnicity, deprivation) which might affect probability of having COPD.

\section{Within the targeted group, active case finding vs opportunistic case finding (individual patient design)}

Baseline characteristics of patients will be compared for each arm (Active and Opportunistic) within each practice to check for balance. Clustering of patients within practices and households (if appropriate) will be accounted for using a multilevel logistic regression model. OR and also RR and risk differences will be estimated [45,46], as will the NNT (Number Needed to Target to identify one additional COPD case). Betweenpractice heterogeneity in the effect of Active versus Opportunistic case finding will be examined, and if present, the range and variability of effects will be appropriately summarized.

\section{Secondary analyses}

In addition the following analyses are also of interest:

- Multilevel logistic regression will be used to compare each of the two components of the Targeted arm with Routine care

- Models will also be extended to estimate the effect of practice-level covariates on rates of COPD detection

- Interaction terms will also be included to assess whether patient-level factors such as age, sex, ethnicity, symptom profile and smoking history modify (interact with) treatment effects.

- The impact of clustering at the household level will be investigated in sensitivity analyses.

\section{Economic analysis}

The trial-based health economic analysis will consider two questions:

How cost-effective is a) an Active approach to casefinding and b) a simple Opportunistic approach compared with routine detection of COPD (routine care), in terms of cost per additional case detected?

A within-trial patient level analysis will be undertaken to determine the total and mean costs of case finding for each trial arm. An incremental cost-effectiveness analysis from a health care perspective will determine the cost per additional case detected, with a sensitivity analysis taking into account patient out of pocket costs in addition to health care costs. True cases of COPD detected by each method will be determined within the trial. The cost of 
case finding and spirometry will be established within the trial, and resource use data will be collected for each method and will include staff time, equipment, diagnostic testing and any consumables required. Data will be collected from a representative sample of practices to ensure generalisability. Unit costs (e.g. staff costs, equipment) will be collected from published sources. In addition a patient cost questionnaire will be administered to all who attend for spirometry to determine their out of pocket (travel and time) costs.

By using data from the trial which uses West Midlands' practices only, there may be limits to the generalisability of the results of the trial. This will be explored within the economic evaluation using extensive sensitivity analysis. Key parameters will be varied to determine the impact of changes on results. Case finding in different patient sub-groups will also be considered. Non-parametric bootstrapping and probabilistic sensitivity analysis will be undertaken to explore uncertainty in the confidence to be placed on the results of the economic analysis and cost effectiveness acceptability curves presented.

\section{Additional analyses}

We also plan further related analyses not detailed here including:

1. A model-based cost-effectiveness analysis to consider longer-term effects of case-detection and subsequent treatment (cost per quality-adjusted life year gained)

2. A qualitative study exploring patients views of case-finding

3. A qualitative study exploring the views of healthcare professionals

\section{Patient advisory group}

A patient advisory group (PAG) has been set up, chaired by $\mathrm{Mr}$ Michael Darby. This group is funded to meet at approximately quarterly intervals or according to need, and will advise on a range of aspects of the design, conduct, analysis and dissemination of the study. The PAG will discuss issues as requested by the CIs and the chair will report their comments back to the investigators.

\section{Data management}

All data will be stored on a password-protected webenabled customized database designed and hosted by the PC-CTRU. Paper-based information will be held in locked filing cabinets in the study office.

\section{Trial steering committee}

We have established a Trial Steering Committee which will meet annually to advise the research team.

Chair: Prof Debbie Jarvis, Imperial

Statistician: Prof Simon Gates, Warwick
Health economist: Dr Jane Wolstenholme, Oxford

Patient representative: Mr Michael Darby

Investigators: Dr Rachel Jordan, Prof David

Fitzmaurice, Prof Peymane Adab.

\section{Data monitoring committee}

This is not a trial of a medicinal product and therefore does not require a data monitoring committee. However, the TSC will undertake some of this role.

\section{Interim analyses \& stopping rules}

There are no planned interim analyses or stopping rules as the study must run for a full calendar year in each general practice in order to allow the opportunistic intervention to occur.

\section{Regulatory issues \\ Ethics approval}

The study has received ethical and research governance approval through the IRAS process. Ref: 11/WM/0403.

\section{Patient consent}

Patients receiving the initial screening respiratory questionnaire will receive a letter of invitation, and patient information leaflet (PIL) (Additional file 1: Appendix 4) and will provide consent by returning their questionnaire with the associated permissions and their contact details. Patients attending spirometry assessment will discuss the trial with the researcher and provide written consent to participate (Additional file 1: Appendix 5). Permission will also be sought to contact patients for future research studies.

\section{Indemnity}

The University of Birmingham holds the relevant insurance policy for this study.

\section{Sponsor}

The University of Birmingham will act as the main sponsor for this study.

\section{Funding}

National Institute for Health Research (NIHR) Programme Grants for Applied Research Programme (Grant Reference Number RP-PG-0109-10061).

Start date: July 2012

Proposed reporting date: July 2015

\section{Publication plan}

The main trial paper reporting the effectiveness and trial-based cost-effectiveness analysis will be submitted initially. 


\section{Discussion}

This trial investigates the effectiveness and costeffectiveness of two alternative modes of systematic case-finding for COPD compared with routine care. Previously undiagnosed patients aged 40 years and over with a positive smoking history will either receive a respiratory questionnaire when they routinely visit their surgery, or will also receive a questionnaire through the post. Those with indicative respiratory symptoms will be offered spirometry to diagnose COPD. There are many strengths of this study including: it is the first of this design based in a large sample of GP practices with a comparator group; it is set in a range of GP practices representative of urban UK; it has a pragmatic design which should reflect real life; the spirometry will be the best quality possible with highly trained staff and quality control; there will be a full cost-effectiveness analysis and also sensitivity analyses to reflect a range of scenarios; the effects will be explored across the range of GPs involved, allowing the cost-effectiveness in different types of GPs to be assessed. This trial should inform practice across the UK and elsewhere with similar healthcare systems, and help to direct current effort towards case-finding more efficiently.

However, a study of this size and complexity has many challenges. We will be coordinating a large number of practices, and as a screening trial, thousands of patients and patient data. Accurately identifying appropriate patients from GP databases with their complex coding is a known problem. It is not known how patients will respond to questionnaires, and whether (or how often) it is practical for GP staff to give out screening questionnaires during routine visits. These are some of the feasibility outcomes we will be measuring. Coordinating and arranging patient assessments with the dual issues of patient and GP capacity will also be a challenge, especially in the short time-frame. And finally, it will be important to discuss how this approach can be rolled out from a trial setting into routine practice.

\section{Additional file}

Additional file 1: Appendix 1. READ codes used for identifying eligible patients. Appendix 2. Screening questionnaire. Appendix 3. Patient costs questionnaire. Appendix 4. Patient Information Sheet. Appendix 5. Patient consent form.

Abbreviations

COPD: chronic obstructive pulmonary disease; NIHR: National Institute for Health Research.

\section{Competing interests}

KKC is affiliated to the Department of General Practice of Peking University Health Sciences Center which receives support from Pfizer for the development of primary care in China.
MM was reimbursed by Boehringer Ingelheim Finland to speak at a $\mathrm{V}$ Scandinavian COPD Research Symposium, Holmenkollen, Oslo, Norway, November 16-17, 2012.

RS received lecture fees from GSK, Grifols, Novartis and Takeda, is a member of advisory boards for GSK, Novartis, Boehringer Ingelheim, Almirall, Takeda Chiesi, Polyphor, CSL Behring, and has received non-commercial grant funding from CSL Behring.

\section{Authors' contributions}

RJ led the design of the trial, with particular contribution from DF and PA, and advice from all other investigators. All investigators contributed to decisions on outcome measures. DF advised on involving general practices, BC and MM advised on lung function testing, SJ designed the health economic analysis section, and RR and JM advised on the statistical aspects. RJ wrote the protocol paper with input from all other authors. All authors have read and approved the final draft

\section{Authors' information}

Principal Investigators: Dr Rachel Jordan, Professor Peymane Adab, Professor David Fitzmaurice.

\section{Acknowledgements}

This paper summarises independent research funded by the National Institute for Health Research (NIHR) under its Programme Grants for Applied Research Programme (Grant Reference Number RP-PG-0109-10061). The views expressed are those of the authors and not necessarily those of the $\mathrm{NHS}$, the NIHR or the Department of Health. TargetCOPD is part of The Birmingham Lung Improvement StudieS - BLISS.

\section{Author details}

${ }^{1}$ School of Health and Populations Sciences, University of Birmingham, Birmingham, UK. ² Lung Investigation Unit, University Hospitals Birmingham NHS Foundation Trust, Birmingham, UK. ${ }^{3}$ Queen Elizabeth Hospital Research Laboratories, Mindelsohn Way, Birmingham, UK. ${ }^{4}$ Business School, University of Birmingham, Birmingham, UK. ${ }^{5}$ Public Health, Epidemiology \& Biostatistics, Public Health Building, School of Health and Population Sciences, University of Birmingham, Edgbaston, Birmingham B15 2TT, UK.

Received: 15 September 2014 Accepted: 26 September 2014

Published: 4 October 2014

\section{References}

1. Halbert RJ, Natoli JL, Gano A, Badamgarav E, Buist AS, Mannino DM: Global burden of COPD: systematic review and meta-analysis. Eur Respir J 2006, 28(3):523-532

2. Buist AS, McBurnie M, Vollmer WM, Gillespie $S$, Burney $P$, Mannino D, Menezes AMB, Sullivan SD, Lee TA, Weiss KB, Jensen RL, Marks GB, Gulsvik A, Nizankowska-Mogilnicka E, on behalf of the BOLD Collaborative Research Group: International variation in the prevalence of COPD (The BOLD Study): a population-based prevalence study. Lancet 2007, 370:741-750.

3. Murray C, Lopez A: Alternative projections of mortality and disability by cause 1990-2020: Global Burden of Disease Study. Lancet 1997, 349:1498-1504.

4. Britton M: The burden of COPD in the U.K.: results from the confronting COPD survey. Respir Med 2003, 97(3):S71-S79.

5. Bednarek M, Maciejewski J, Wozniak M, Kuca P, Zielinski J: Prevalence, severity and underdiagnosis of COPD in the primary care setting. Thorax 2008, 63(5):402-407.

6. Mannino DM, Gagnon RC, Petty TL, Lydick E: Obstructive Lung Disease and Low Lung Function in Adults in the United States: Data From the National Health and Nutrition Examination Survey, 1988-1994. Arch Intern Med 2000, 160(11):1683-1689.

7. Shahab L, Jarvis MJ, Britton J, West R: Prevalence, diagnosis and relation to tobacco dependence of chronic obstructive pulmonary disease in a nationally representative population sample. Thorax 2006, 61(12):1043-1047.

8. US Preventive Services Task Force: Screening for Chronic obstructive pulmonary disease using spirometry: US Preventive Services Task Force Recommendation Statement. Ann Intern Med 2008, 148:529-534.

9. Soriano JB, Zielinski J, Price D: Screening for and early detection of chronic obstructive pulmonary disease. Lancet 2009, 374(9691):721-732. 
10. Jones RC, Price D, Ryan D, Sims EJ, von Ziegenweidt J, Mascarenhas L, Burden A, Halpin DMG, Winter R, Hill S, Kearney M, Holton K, Moger A, Freeman D, Chisholm A, Bateman ED, on behalf of The Respiratory Eff ectiveness Group: Opportunities to diagnose chronic obstructive pulmonary disease in routine care in the UK: a retrospective study of a clinical cohort. The Lancet Respiratory Medicine 2014, 2(4):267-276.

11. On the state of the Public Health: Annual report of the Chief Medical Officer 2004. Available from: http://webarchive.nationalarchives.gov.uk/ 20130107105354/http://www.dh.gov.uk/prod_consum_dh/groups/ dh_digitalassets/@dh/@en/documents/digitalasset/dh_4115781.pdf 2009.

12. Healthcare Commission: Clearing the Air. A National Study of Chronic Obstructive Pulmonary Disease. 2006. http://webarchive.nationalarchives.gov. uk/20090724223245/http://www.library.nhs.uk/RESPIRATORY/ViewResource. aspx?resID=144395.

13. An outcomes strategy for people with chronic obstructive pulmonary disease (COPD) and asthma in England. http://www.dh.gov.uk/en/ Publicationsandstatistics/Publications/PublicationsPolicyAndGuidance/ DH_127974.

14. British Lung Foundation: Invisible lives. Chronic obstructive pulmonary disease (COPD) - finding the missing millions. Available from: http://www.blf.org.uk/ Page/Special-Reports 2008.

15. Mapel DW, Frost F, Hurly J, Petersen H, Roberts M, Marton J, Shah H: An algorithm for the identification of undiagnsoed COPD cases using administrative claims data. J Manag Care Pharm 2006, 12(6):458-465.

16. Soljak M, Flowers J: Closing the gap. Using prevalence models for long-term conditions in the United Kingdom. J Ambulatory Care Manage 2008, 31(3):211-215.

17. Nottinghamshire County Teaching PCT: Search to find COPD 'Missing Millions'. 2007. Last accessed 2008

18. Sandwell NHS Primary Care Trust: Finding the Missing Millions. Sandwell Health Report 2008, 1-2. Last accessed 2008.

19. Frank $T$, Hazell M, Linehan M, Frank $P$ : The diagnostic accuracies of chronic obstructive pulmonary disease (COPD) in general practice: the results of the MAGIC (Manchester Airways Group Identifying COPD) study. Prim Care Resp J 2006, 15:286-293.

20. Tinkelman D, Price D, Nordyke R, Halbert R: COPD screening efforts in primary care: what is the yield? Prim Care Resp J 2007, 16(1):41-48.

21. Buffels J, Degryse J, Heyrman J, Decramer M: Office Spirometry Significantly Improves Early Detection of COPD in General Practice. Chest 2004, 125(4):1394-1399.

22. Gingter C, Wilm S, Abholz HH: Is COPD a rare disease? Prevalence and identification rates in smokers aged 40 years and over within general practice in Germany. Fam Pract 2009, 26(1):3-9.

23. van Schayck CP, Loozen JMC, Wagena $E$, Akkermans RP, Wesseling GJ: Detecting patients at a high risk of developing chronic obstructive pulmonary disease in general practice: cross sectional case finding study. BMJ 2002, 324(7350):1370.

24. Vandevoorde J, Verbanck S, Gijssels L, Schuermans D, Devroey D, De Backer J, Kartounian J, Vincken W: Early detection of COPD: A case finding study in general practice. Respir Med 2007, 101(3):525-530.

25. Kelly JL, Elkin SL, Fluxman J, Polkey MI, Soljak MA, Hopkinson NS: Breathlessness and Skeletal Muscle Weakness in Patients Undergoing Lung Health Screening in Primary Care. COPD 2013, 10:40-54. doi:10.3109/15412555.2012.727923.

26. Dirven JAM, Tange HJ, Muris JWM, van Haaren KMA, Vink G, van Schayck OCP: Early detection of COPD in general practice: patient or practice managed? A randomised controlled trial of two strategies in different socioeconomic environments. Prim Care Respir J 2013, 22(3):331-337.

27. Haroon S, Adab P, Griffin C, Jordan R: Case finding for chronic obstructive pulmonary disease in primary care: a pilot randomised controlled trial. Brit J Gen Pract 2013, 63(606):26-27.

28. The UK NSC policy on Chronic Obstructive Pulmonary Disease. http://www.screening.nhs.uk/copd.

29. Wilt TJ, Niewoehner D, MacDonald R, Kane RL: Management of Stable Chronic Obstructive Pulmonary Disease: A Systematic Review for a Clinical Practice Guideline. Ann Intern Med 2007, 147(9):639-653.

30. Wilt T, Kim C-B, Kane R, Linabery A, Tacklind J, MacDonald R, Rutks I: Use of spirometry for case finding, diagnosis and management of chronic obstructive pulmonary disease (COPD). Evidence Report/Technology assessment No 121 (prepared by the Minnesota Evidence-based practice Center under Contract No
290-02-0009) 2005;AHRQ Publication No. 05-E017-2. Rockville, MD: Agency for Healthcare Research and Quality; 2005

31. National Institute for Health and Clinical Excellence: Chronic obstructive pulmonary disease: management of chronic obstructive pulmonary disease in adults in primary and secondary care. London: National Institute for Health and Clinical Excellence; 2010.

32. Jordan RE, Lam K-BH, Cheng KK, Miller MR, Marsh JL, Ayres JG, Fitzmaurice $D$, Adab P: Case finding for chronic obstructive pulmonary disease: a model for optimizing a targeted approach. Thorax 2010, 65:492-498.

33. Decramer M, Celli B, Kesten S, Lystig T, Mehra S, for the UPLIFT investigators, Tashkin DP: Effect of tiotropium on outcomes in patients with moderate chronic obstructive pulmonary disease (UPLIFT): a prespecified subgroup analysis of a randomised controlled trial. Lancet 2009, 374(9696):1171-1178.

34. Hazell ML, Morris JA, Linehan MF, Frank PI, Frank TL: Factors influencing the response to postal questionnaire surveys about respiratory symptoms. Prim Care Respir J 2009, 18(3):165-170. doi:10.3132/pcrj.2009.00001.

35. Miller MR, Hankinson J, Brusasco V, Burgos F, Casaburi R, Coates A, Crapo R, Enright $P$, van der Grinten CP, Gustafsson P, Jensen R, Johnson DC, MacIntyre N, McKay R, Navajas D, Pedersen OF, Pellegrino R, Viegi G, Wanger J, ATS/ERS Task Force: Standardisation of spirometry. Eur Respir J 2005, 26(2):319-338.

36. EQ-5D. http://www.euroqol.org/home.html.

37. Jones PW: Development and first validation of the COPD Assessment Test. Eur Respir J 2009, 34(3):648-654.

38. Raab GM, Butcher I: Balance in cluster randomized trials. Stat Med 2001, 20:351-365

39. Carter B, Hood K: Balance algorithm for cluster randomised trials. BMC Med Res Methodol 2008, 8:65.

40. Quality and Outcomes Framework. http://www.hscic.gov.uk/qof.

41. Quanjer PH, Stanojevic S, Cole TJ, Baur X, Hall GL, Culver BH, Enright PL, Hankinson JL, Ip MSM, Zheng J, Stocks J, and the ERS Global Lung Function Initiative: Multi-ethnic reference values for spirometry for the 3-95-yr age range: the global lung function 2012 equations. Eur Respir J 2012, 40:1324-1343. doi:10.1183/09031936.00080312.

42. Simpson CR, Hippisley-Cox J, Sheikh A: Trends in the epidemiology of chronic obstructive pulmonary disease in England: a national study of 51804 patients. Br J Gen Pract 2010, 60(576):277-284.

43. Adams G, Gulliford MC, Ukoumunne OC, Eldridge S, Chinn S, Campbell MJ: Patterns of intra-cluster correlation from primary care research to inform study design and analysis. J Clin Epidemiol 2004, 57(8):785-794.

44. Gulliford MC, Adams G, Ukoumunne OC, Latinovic R, Chinn S, Campbell MJ: Intraclass correlation coefficient and outcome prevalence are associated in clustered binary data. J Clin Epidemiol 2005, 58:246-251.

45. Austin PC: Absolute risk reductions, relative risks, relative risk reductions, and numbers needed to treat can be obtained from a logistic regression model. J Clin Epidemiol 2010, 63(1):2-6.

46. Lacalio AR, Margolis DJ, Berlin JA: Relative risks and confidence intervals were easily computed indirectly from multivariable logistic regression. J Clin Epidemiol 2007, 60(9):874-882.

doi:10.1186/1471-2466-14-157

Cite this article as: Jordan et al:: TargetCOPD: a pragmatic randomised controlled trial of targeted case finding for COPD versus routine practice in primary care: protocol. BMC Pulmonary Medicine 2014 14:157. 